\title{
NMMHC-IIA-dependent nuclear location of CXCR4 promotes migration and invasion in renal cell carcinoma
}

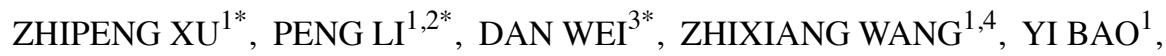 \\ JIPENG SUN $^{6}$, LE QU ${ }^{1,5}$ and LINHUI WANG ${ }^{1}$ \\ ${ }^{1}$ Department of Urology, Changzheng Hospital, The Second Military Medical University, Shanghai 200001; \\ ${ }^{2}$ Division of Urology, The 153rd Hospital of Chinese Liberation Army, Zhengzhou, Henan 450000; \\ ${ }^{3}$ Division of Endocrinology, Department of Internal Medicine, Shandong Provincial Qianfoshan Hospital, Shandong \\ University, Jinan, Shandong 250013; ${ }^{4}$ Department of Urology, The 458th Hospital of PLA, Guangzhou 510602; \\ ${ }^{5}$ Department of Urology, Nanjing General Hospital of Nanjing Military Command, Nanjing 210002; \\ ${ }^{6}$ Health Contingent, No. 71210 Unit of People's Liberation Army, Yantai, Shantong 264001, P.R. China
}

Received March 11, 2016; Accepted July 18, 2016

DOI: $10.3892 /$ or.2016.5082

\begin{abstract}
The chemokine receptor cysteine (C)-X-C receptor (CXCR4) is a G-protein-coupled receptor that exerts a vital role in distant metastasis of renal cell carcinoma (RCC). Emerging evidence demonstrates that CXCR4 as the cytomembrane receptor translocated into the nucleus to facilitate cell migration and, therefore, determine the prognosis of several types of malignancies. However, the biological mechanism of nuclear location of CXCR4 remains unclear. In the present study, we confirmed the significant implications of the putative nuclear localization sequence (NLS) '146RPRK149' on CXCR4 subcellular localization and metastatic potential by point-mutation assay in RCC cell lines. Importantly, mass spectrum followed by immunoprecipitation identified non-muscle myosin heavy chain-IIA (NMMHC-IIA) as the CXCR4-interacting protein. Furthermore, pharmaceutical inhibition of NMMHC-IIA by blebbistatin dampened the nuclear translocation of CXCR4 as well as the metastatic capacity of RCC cells. In conclusion, the present study may drive the comprehensive progress toward elucidating the mechanism responsible for CXCR4 nuclear function and metastasis in tumors.
\end{abstract}

Correspondence to: Dr Linhui Wang or Dr Le Qu, Department of Urology, Changzheng Hospital, Second Military Medical University, 415 Fengyang Road, Shanghai 200001, P.R. China

E-mail: wanglinhuicz@163.com

E-mail: septsoul@hotmail.com

${ }^{*}$ Contributed equally

Abbreviations: CXCR4, cysteine (C)-X-C receptor 4; $\mathrm{RCC}$, renal cell carcinoma; NMMHC-IIA, non-muscle myosin heavy chain-IIA; GPCR, G-protein coupled receptor

Key words: CXCR4, renal cell carcinoma, nuclear location, NMMHC-IIA, gene mutations

\section{Introduction}

Renal cell carcinoma (RCC) is the fifth most common cancer worldwide, accounting for 2-3\% of all malignant diseases in adults $(1,2)$. Despite the fact that RCC is frequently diagnosed at a small and early stage, there are approximately one third of patients with renal cancer presenting metastatic diseases at the time of diagnosis and 30-40\% of patients with localized renal cancer develop metastasis after surgery, which is the major clinical challenge in RCC (3-5). RCC tumorigenesis and tumor progression comprises diverse molecules and mechanisms. It has been suggested that the directional trafficking play crucial roles in driving the tumor metastasis $(6,7)$.

CXCR4 chemokine receptor belongs to the group of seven transmembrane G-protein coupled receptors (GPCR) which was found in more than 20 types of tumors in human, including prostate, ovarian and esophageal cancer, melanoma and RCC (8-13). Growing evidence indicated that high level of CXCR4 was significantly implicated in tumorigenesis and metastasis in many malignancies $(14,15)$. Previous studies have shown that nuclear localized CXCR4 determined prognosis for colon, gastric, lung and colorectal cancer (16-19). While in different types of tumors, the prognostic prediction of nuclear localized CXCR4 was different, even opposite. Nuclear CXCR4 expression was associated with a favorable prognosis in non-small cell lung cancer (17), but a poor prognosis in primary colon cancer $(20,21)$. Our previous study confirmed that high level of CXCR4 was associated with poor overall and recurrence-free survival in RCC patients (22), and nuclear translocated CXCR4 may play functional roles in metastatic RCC (23). Thus, considerable attention has been focused on the mechanism of the nuclear translocation of CXCR4, which may promote tumor growth and metastasis. However, detailed mechanisms are still to be illustrated.

The surface-to-nucleus signaling pathways in a cascade (Ras/MAP kinase) or receptor-activated proteins acting singly (STATs) have been well-recognized. However, the ability of cytomembranous receptors to directly translocate 
to the nucleus and influence on the cellular functions is less investigated. While a great number of nuclear translocated cytomembranous receptors have been reported, only a few cases (Notch, APP and ErbB4) have been shown to change nuclear function convincingly (24-26). In addition, preliminary evidence showed that CXCR4 may be one of the candidates.

Almost every plasm-nucleolus shuttled protein harbor a functional nuclear localization sequence (NLS) or bind to transport proteins which possess an NLS. Our previous study showed that CXCR4 contained an NLS located in amino acids 90-170, which was very long and not precise (23). Furthermore, NLS '146RPRK149', within CXCR4 presumed by PSORTII (http://psort.nibb.ac.jp/) has been identified to contribute to nuclear localization in prostate cancer cells (27). Whether this putative NLS also modulates nuclear translocation of CXCR4 in RCC remains unknown. The present study investigated the function of nuclear localized CXCR4 and its biological mechanisms in RCC cells.

\section{Materials and methods}

Cell lines and culture conditions. Human RCC cell lines (A498 and ACHN) were obtained from the Chinese Academy of Sciences (Shanghai, China). The cells were incubated in Roswell Park Memorial Institute-1640 medium containing $10 \%$ heat-inactivated fetal bovine serum and antibiotics (100 $\mu \mathrm{g} / \mathrm{ml}$ of penicillin-streptomycin). Cells were grown as a monolayer on plastic cell culture dishes at $37^{\circ} \mathrm{C}$ in a humidified atmosphere containing $5 \% \mathrm{CO}_{2}$.

Lentiviral vectors and infection. The lentivirus encoding EGFP, EGFP-CXCR4 or EGFP-CXCR4MutNLS (combination of R146A, R148A and R149A point mutations within the NLS) plasmids were packaged and purified at HanBio Biotechnology (Shanghai, China) and infected cells following the manufacturer's instructions.

Subcellular fractionation and western blot analysis. RCC cells were serum-starved for $24 \mathrm{~h}$. Subcellular fractionations were performed by Nuclear/Cytosol Fractionation kit (BioVision, San Francisco, CA, USA) following the manufacturer's instructions. Western blotting was performed as previously described (23) with anti-human CXCR4 antibody (1:1,000), anti-topoisomerase I (1:1,000) (both from Santa Cruz Biotechnology, Santa Cruz, CA, USA) and anti-CD44 (1:1,000; Cell Signaling, BSN, USA) antibodies.

Point mutation. Combination of R146A, R148A and R149A point mutations within the NLS of GFP-CXCR4 fusion protein were generated using the gene splicing by overlap extension-PCR, SOE-PCR; pEGFPN1-CXCR4 acted as the template (23). The forward and reverse primers purchased from Sango Biotech (Shanghai, China), were: i) Rm689 F, TAGA CCACCTTTTCAGCCAACAGCGCCGCTGGCGCCTGA CTGTTGGTGGCGTGGACGA and R, AAAGCTTGCTG GAGTGAAAACTTGAAGAC. The resultant plasmids were pEGFPN1-CXCR4NLS689. Positive CXCR4 mutant clones were selected with ampicillin and further purified by Maxiprep (Omega Bio-Tek, Norcross, GA, USA). Accuracy of the mutations was confirmed by Sangon Biotech (Shanghai, China).
Cell Counting Kit-8 (CCK-8) assay. Cells were seeded into 96 -well culture plates $\left(5 \times 10^{3}\right.$ cells/well $)$. At indicated time, $10 \mu \mathrm{l}$ CCK-8 reagent (Dojindo Molecular Technologies, Inc., Kumamoto, Japan) was added to each well and incubated for $2 \mathrm{~h}$ at $37^{\circ} \mathrm{C}$. Absorbance values at a wavelength of $450 \mathrm{~nm}$ were recorded using a microplate reader (Varioskan Flash; Thermo Scientific, Waltham, MA, USA). Viability (\%) was calculated based on the optical density (OD) values, as follows: (OD of time sample - blank)/(OD of control sample - blank) x 100.

Scratch assay. When cells reached $90 \%$ confluency, a scratch was made through each well using a sterile pipette tip. Cells were monitored under a microscope (magnification, x50) for indicated time after wounding.

Transwell invasion and migration assays. The invasion and migration abilities were performed with the filters (Corning, Lowell, MA, USA) and Transwells (Millipore, Billerica, MA, USA) following the manufacturer's instructions. In the invasion assay cells were stained with $0.5 \%$ crystal violet, while migrated cells were counted using 4',6-diamidino-2-phenylindole (DAPI).

Immunofluorescence and confocal microscopy. LV-EGFPCXCR4 and LV-EGFP-CXCR4MutNLS infected RCC cells were evaluated by immunofluorescence as previously described (23). The coverslips were viewed under a fluorescence microscope (Nikon C1-i; Nikon, Tokyo, Japan) or a Leica Microsystems SP5 confocal microscope.

Immunoprecipitation. The immunoprecipitation of CXCR4 was performed by pull-down with CXCR4 antibody from total protein lysates according to the manufacturer's protocol (Invitrogen, Karlsruhe, Germany). The CXCR4-pull-down products were subjected to $10 \%$ denaturing polyacrylamide gel electrophoresis and visualized by silver staining. The single protein band of $230 \mathrm{kDa}$ was analyzed using MS/MS spectra and the results were confirmed by western blot analysis.

RCC tissue. We obtained human RCC surgical resection samples at the Department of Pathology, Changhai Hospital (Shanghai, China). The samples originated from one patient with RCC, who provided written informed consent to use the specimens. The study design was approved by the Changhai Hospital Ethics Committee.

Immunofluorescent localization. Co-localization of NMMHC-IIA and CXCR4 in RCC tissue was observed by immunofluorescence (IF). The sections were prepared following the instructions of the manufacturer, and the slides were double-stained with the primary antibodies, a mouse antibody specific to CXCR4 and a rabbit antibody specific to NMMHC-IIA, respectively.

Statistical analysis. GraphPad Prism version 6.0 (GraphPad, San Diego, CA, USA) was used for all statistical analyses. Data are presented as the means \pm standard deviation from at least three separate experiments. The t-test (two-tailed) was used to 
A

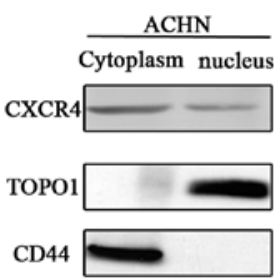

C

A498 EGFP-CXCR4

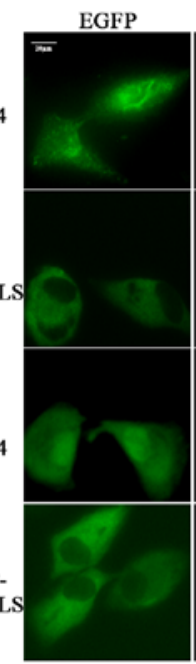

E

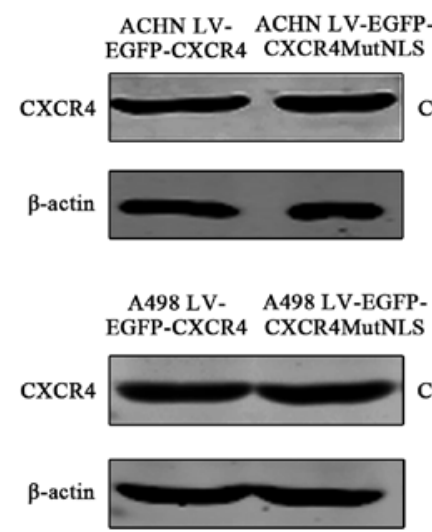

ACHN EGFPCXCR4MutNI
A498

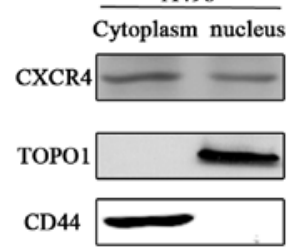

DAPI Merge

D

B
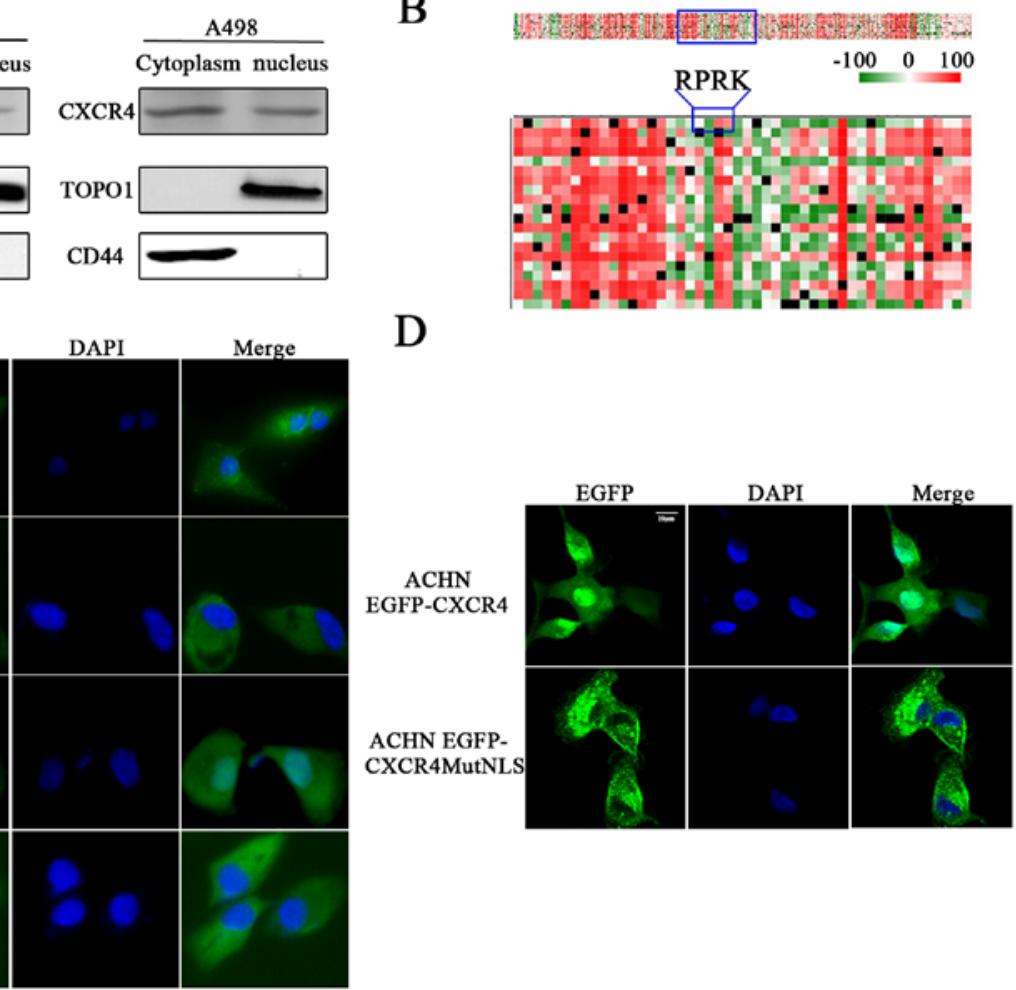

ACHN LV- ACHN LV-EGFP$\begin{gathered}\text { EGFP-CXCR4 } \\ \text { cytoplasm }\end{gathered}$
$\begin{gathered}\text { CXCR4MutNLS } \\ \text { cytoplasm }\end{gathered}$ ACHN LV- ACHN LV-EGFPEGFP-CXCR4 CXCR4MutNLS CXCR4

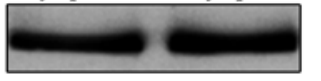

CXCR4 nucleus nucleus

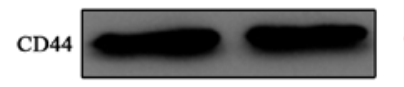

TOPO1
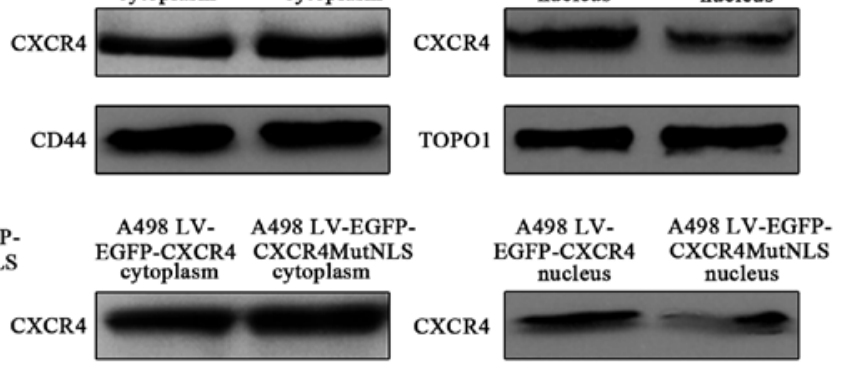

CD44

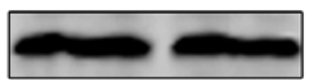

TOPO1

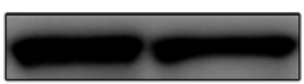

Figure 1. The NLS sequence at 146RPRK149 is of great value in CXCR4 nuclear localization. (A) The fractionation of cells demonstrated that CXCR4 was present in both the cytoplasm and nucleus [topoisomerase I (TOPO1), nuclear control; CD44, cytoplasmic control]. (B) PredictProtein software analysis showed the scores when the amino acid was mutated to alanine in the NLS of CXCR4: 146R mutated to A scored as -4; 148R mutated to A scored 59 and $149 \mathrm{R}$ mutated to A scored 46. (C) Immunofluorescence was performed in EGFP-CXCR4 and EGFP-CXCR4MutNLS (146, 148 and 149 amino acids were mutated to alanine in the NLS of CXCR4) transfected cells. The cell nuclei were counted by DAPI and the scale bar is $20 \mu \mathrm{m}$. (D) The CXCR4 distribution in EGFP-CXCR4 and EGFP-CXCR4MutNLS transfected cells were detected by confocal microscopy and the scale bar is $20 \mu \mathrm{m}$. (E) The fractionation of LV-EGFP-CXCR4 and LV-EGFP-CXCR4MutNLS RCC cells were prepared and subjected to western blot analysis cytoplasmic and nuclear distribution of CXCR4.

draw a comparison between groups, and the significance level was set at $\mathrm{P}<0.05$.

\section{Results}

NLS mutation within CXCR4 inhibits its nuclear localization in RCC cells. We have previously found that CXCR4 protein was positive in A498 and ACHN cells lines (data not shown). To confirm the subcellular localization of CXCR4, RCC cells were fractionated into nuclear and cytoplasmic fractions, and the purity of subcellular fractions was confirmed by expression of CD44, a non-nuclear control (28) and topoisomerase 1, a nuclear control (29) (Fig. 1A). We found that A498 and
ACHN expressed CXCR4 in both the nuclear and cytoplasmic fractions, which suggested that CXCR4 was expressed in the cytoplasm, but also within the nucleus of RCC cells.

Plasm-nucleolus shuttled proteins often contain a functional NLS or bind to transport proteins possessing an NLS (30). Our previous study suggested that the NLS of CXCR4 was located in amino acids 90-170, but it was not accurate (23). A bioinformatics analysis using the PSORTII NLS prediction software revealed a putative NLS, 'RPRK' $(27,31)$ between amino acids 146-149 within CXCR4 amino acid sequence. To determine whether the putative NLS, '146RPRK149', was functional and contributed to nuclear translocation of CXCR4, we evaluated the subcellular distribution of wild-type EGFP-CXCR4, 

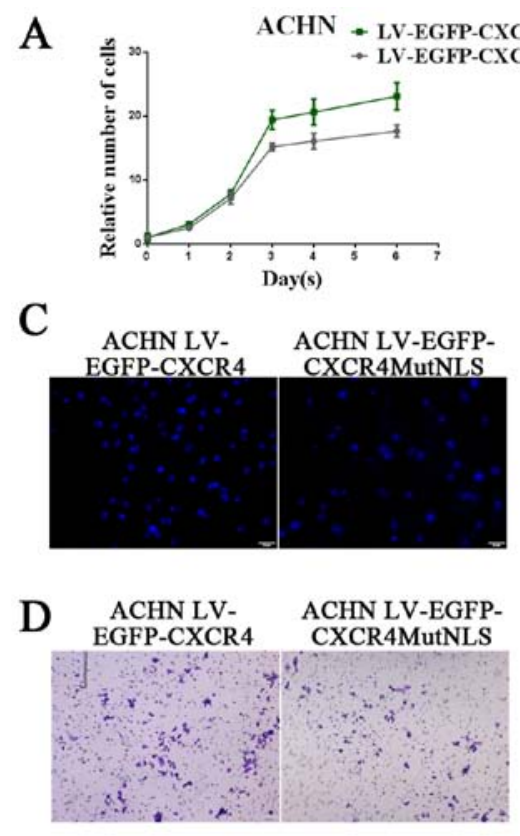

B

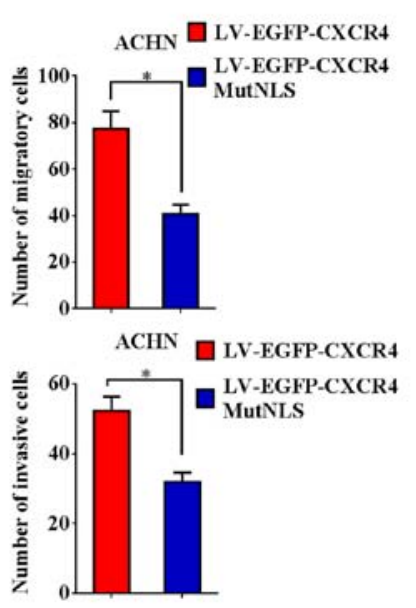

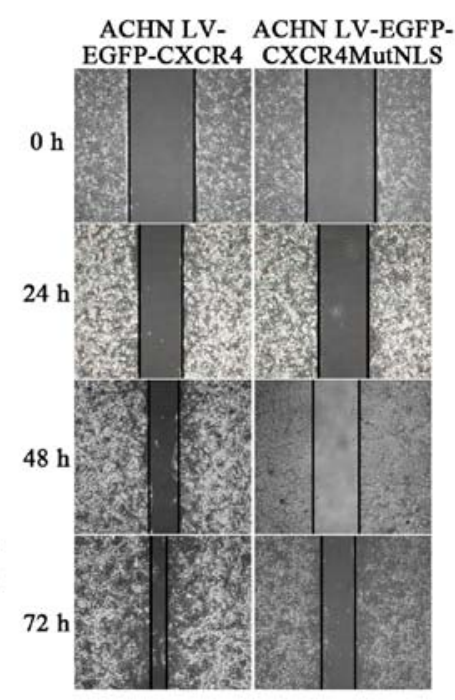

Figure 2. CXCR4 in the nucleus promotes the proliferation, migration and invasion of RCC cells. (A) CCK-8 assay of LV-EGFP-CXCR4 and LV-EGFP-CXCR4MutNLS infected ACHN cells at indicated time. (B) Wound-healing experiments of LV-EGFP-CXCR4 and LV-EGFP-CXCR4MutNLS infected ACHN cells were performed and the migration distances were measured at indicated time. LV-EGFP-CXCR4 and LV-EGFP-CXCR4MutNLS infected ACHN cells were detected by (C) Transwell migration and (D) invasion assays and the scale bar is $100 \mu \mathrm{m}$; ${ }^{*} \mathrm{P}<0.05,{ }^{* *} \mathrm{P}<0.01$.

three mutational fusion proteins in which arginine 146,148 and 149 were separately mutated to an alanine (CXCR4R146A, CXCR4P148A and CXCR4R149A, respectively), as well as a fusion protein where three arginine 146, 148 and 149 in the NLS were all mutated to alanines (CXCR4MutNLS) by PredictProtein software (Fig. 1B). Plasmids encoding EGFPCXCR4 were transfected into ACHN cells and examined by immunofluorescence microscopy. Although EGFP-CXCR4 was localized in both cytoplasm and nucleus (Fig. 1C), CXCR4R146A, CXCR4R148A and CXCR4R149A were also detectable at the nucleus, suggesting that R146A, R148A or R149A mutation were insufficient to inhibit CXCR4 localization to nucleus (data not shown). However, we detected that CXCR4 diffusely throughout the cytoplasm upon transfection of EGFP-CXCR4 plasmid, but CXCR4 in the nucleus of EGFP-CXCR4MutNLS transfected cells was obviously decreased (Fig. 1C). The confocal microscopy further precisely confirmed that, compared with EGFP-CXCR4 group, the nuclear distribution of CXCR4 was obviously less in EGFP-CXCR4MutNLS transfected ACHN cells (Fig. 1D). Additionally, to confirm that CXCR4 was decreased in the nucleus, LV-EGFP-CXCR4 or LV-EGFP-CXCR4MutNLS infected RCC cells were fractionated into nuclear and cytoplasmic fractions for western blot analysis (Fig. 1E). Consistent with IF observations, we found that cells infected with wildtype LV-EGFP-CXCR4 and LV-EGFP-CXCR4MutNLS were both presented with cytoplasmic CXCR4 detection, while the nuclear CXCR4 was significantly reduced in LV-EGFPCXCR4MutNLS infected RCC cells. Collectively, these data suggest that the '146RPRK149' may be involved in localization of CXCR4 to the nucleus in RCC cells and may help us further investigate the function of nuclear CXCR4.
CXCR4 in the nucleus promotes proliferation, migration and invasion of RCC cells. CCK-8 assays were performed to investigate the effects of nuclear CXCR4 on proliferation of ACHN cell line. Compared with LV-EGFP-CXCR4MutNLS infected group, LV-EGFP-CXCR4 infected ACHN cells had a significant growth promoting capacity (Fig. 2A) indicating that high level of CXCR4 in the nucleus promoted RCC cell growth. To further investigate the function of CXCR4 in the nucleus, the migration ability of ACHN cell was assessed by scratchwound and Transwell assays. The scratch-wound assay showed that the migration ability of the LV-EGFP-CXCR4MutNLS infected ACHN cells was lower than the LV-EGFP-CXCR4 infected ACHN cells. We found that the cell-free area of the LV-EGFP-CXCR4 group was obviously narrower than the LV-EGFP-CXCR4MutNLS group at 24, 48 and $72 \mathrm{~h}$ after drawing the 'scratch' line on the monolayer cells (Fig. 2B) and confirmed the results by Transwell migration assay (Fig. 2C). In Transwell invasion assays, the number of invaded cells stained with crystal violet was significantly higher in the LV-EGFP-CXCR4 group (Fig. 2E). These results showed that CXCR4 in the nucleus can promote the proliferation, migration and invasion ability of RCC cells.

Nuclearlocalization ofCXCR4 partlydepends on NMMHC-IIA. Next, we investigated whether CXCR4 specifically bound intracellular partners that affected its subcellular localization. Immunoprecipitation with cell lysates of ACHN cells (Fig. 3A) revealed the presence of protein bands of spots (Fig. 3A-A-D) that were specifically associated with CXCR4. We focused on the spots $\mathrm{D}(230 \mathrm{kDa})$, the bands were excised, digested with trypsin, and the peptides were analyzed by mass spectrometry. The $230 \mathrm{kDa}$ band was unequivocally identified as non-muscle 
A

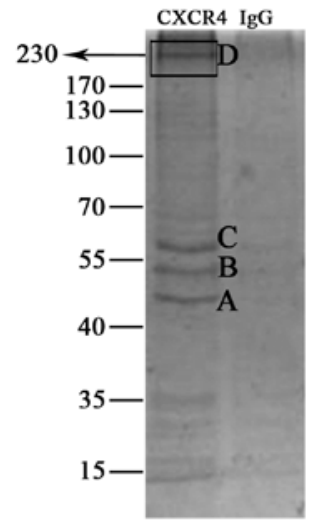

$\mathrm{D}$

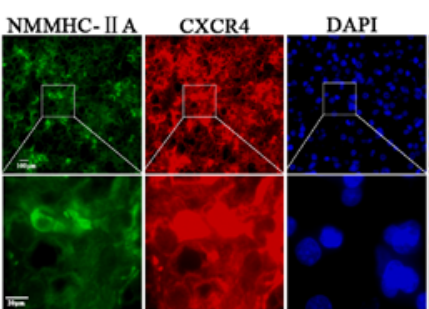

B

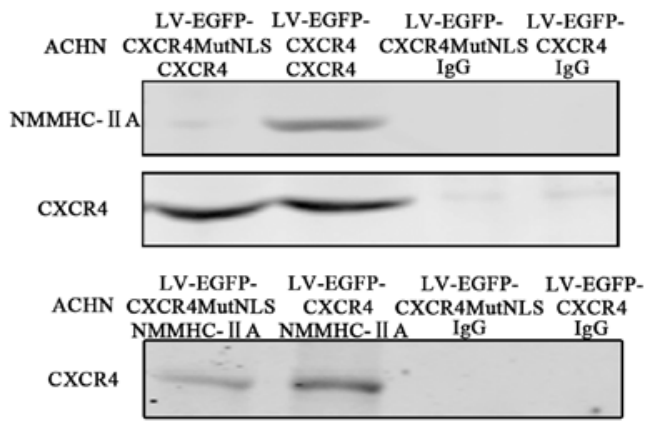

NMMHC-II

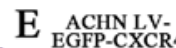

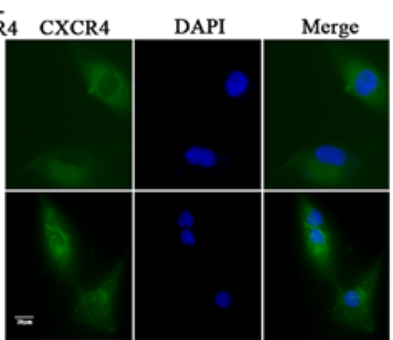

Figure 3. Nuclear localization of CXCR4 and its biological functions partly depend on NMMHC-IIA. (A) ACHN cell lysates were prepared and subjected to immunoprecipitation with CXCR4 and IgG antibody. The eluted proteins were separated by SDS-PAGE and visualized by silver staining. (B and C) CXCR4 binding lysates and NMMHC-IIA binding lysates of LV-EGFP-CXCR4 and LV-EGFP-CXCR4MutNLS ACHN cells were subjected to western blot analysis of NMMHC-IIA and CXCR4. (D) The CXCR4 (red) and NMMHC-IIA (green) was visualized by immunofluorescence staining in human RCC tissue. The cell nuclei were counted by DAPI and scale bar was $20 \mu \mathrm{m}$. (E) EGFP-CXCR4 (green) and nuclei (blue) were counterstained with DAPI after induced with blebbistatin $(50 \mu \mathrm{M}, 24 \mathrm{~h})$. Right panels showed merged fluorescence microscopic images.

A

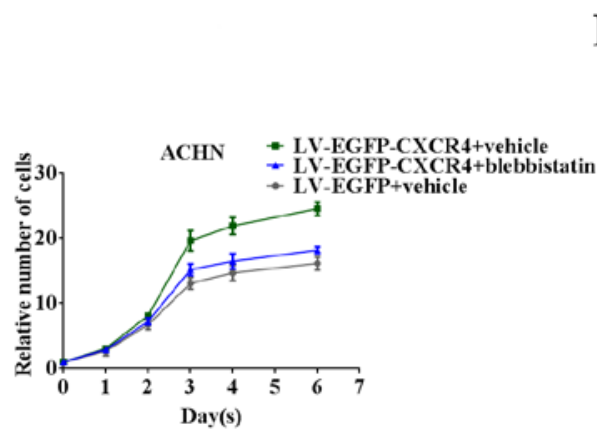

C

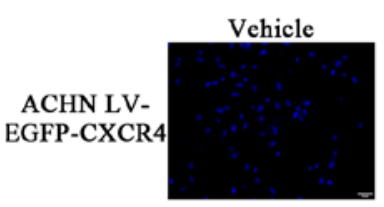

D

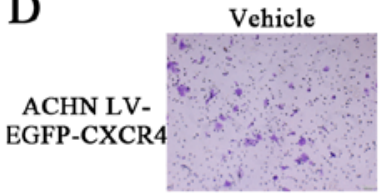

B ACHN LV-EGFP-CXCR4

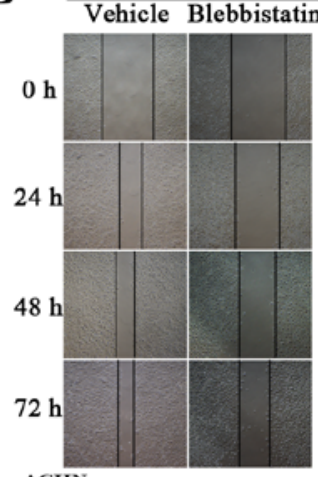

ACHN $\square$ Vehicle

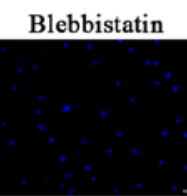

Blebbistatin

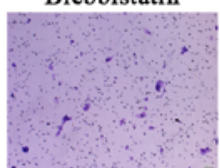

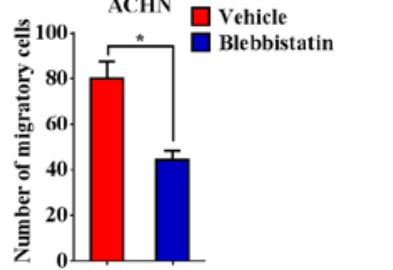

ACHN

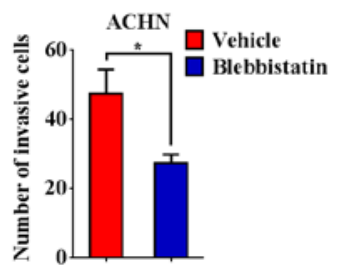

Figure 4. Inhibition of NMMHC-IIA suppresses the RCC progression. (A) CCK-8 assay of LV-EGFP-CXCR4 infected ACHN cells co-incubated with blebbistatin $(50 \mu \mathrm{M})$ at indicated time. (B) Wound-healing experiment of blebbistatin $(50 \mu \mathrm{M})$ induced LV-EGFP-CXCR4 infected ACHN cells at indicated time. The migration distances were measured and analyzed by comparing two independent groups. (C) Transwell migration assay of LV-EGFP-CXCR4 infected ACHN cells treated with blebbistatin $(50 \mu \mathrm{m})$ or vehicle for $24 \mathrm{~h}$ and the scale bar was $100 \mu \mathrm{m}$. (D) Transwell invasion assay of LV-EGFP-CXCR4 infected ACHN cells treated with blebbistatin $(50 \mu \mathrm{m})$ or vehicle for $24 \mathrm{~h}$ and the scale bar was $100 \mu \mathrm{m}$; ${ }^{*} \mathrm{P}<0.05,{ }^{* *} \mathrm{P}<0.01$. 
Table I. Mass spectrometry analysis of the $230 \mathrm{kDa}$ band.

\begin{tabular}{lcrrlr}
\hline Groups & Protein mass (Da) & PepCount & Unique PepCount & \multicolumn{1}{c}{ Sequence header } & Relative abundance (\%) \\
\hline 1 & $226,532.68$ & 34 & 23 & Non-muscle myosin IIA & 13.62 \\
2 & $85,015.53$ & 6 & 4 & Raichu-404X & 5.54 \\
3 & $39,745.56$ & 3 & 2 & Cysteine (C)-X-C receptor 4 & 5.68 \\
4 & $14,728.26$ & 1 & 1 & Ubiquitin-60S ribosomal protein L40 & 7.03 \\
5 & $83,673.87$ & 1 & 1 & Protein kinase CE & 0.81 \\
6 & $16,387.7$ & 1 & 1 & Cystatin-SN & 12.06 \\
\hline
\end{tabular}

myosin IIA by MS/MS analysis (Table I). To confirm the identity of the $230 \mathrm{kDa}$ protein, western blot analysis with an antibody against NMMHC-IIA and CXCR4 were performed in CXCR4 or NMMHC-IIA binding protein lysates of LV-EGFP-CXCR4 and LV-EGFP-CXCR4MutNLS infected ACHN cells (Fig. 3B and C), which showed that CXCR4 and NMMHC-IIA could bind with each other mutually. Additionally, colocalization pattern of NMMHC-IIA and CXCR4 was presented by immunofluorescence in RCC tissue (Fig. 3E). The result showed that the distribution of CXCR4 and NMMHC-IIA were almost overlapping, which further revealed that NMMHC-IIA specifically bound with CXCR4.

To assess the involvement of NMMHC-IIA in the subcellular localization of CXCR4, blebbistatin, a specific inhibitor of NMMHC-IIA, was used to inhibit NMMHC-IIA activation, and CXCR4 nucleus localization was observed by IF. Treatment with $50 \mu \mathrm{M}$ blebbistatin, CXCR4 in the nucleus was significantly decreased in LV-EGFP-CXCR4 infected ACHN cells (Fig. 3F). These results suggested that targeting NMMHC-IIA interfered with CXCR4 subcellular localization.

Inhibition of NMMHC-IIA suppresses the metastatic potential of RCC cells. Then, the functional relationship between NMMHC-IIA and CXCR4 was analyzed. Blebbistatin suppressed the growth ability of LV-EGFP-CXCR4 infected ACHN cells (Fig. 4A). We also found that blebbistatin decreased the migratory and invasive ability in LV-EGFP-CXCR4 infected ACHN cells (Fig. 4B-D). Collectively, these results indicated that inhibition of NMMHC-IIA suppressed the growth, migration and invasion capacity of RCC cells by inhibiting the nuclear location of CXCR4.

\section{Discussion}

CXCR4 has been identified as a key factor involving in organ-specific metastasis of RCC (32). Our previous study observed that nuclear localization of CXCR4 was found in metastatic but not primary RCC lesions (33). It is known that within thousands of cancer cells, just a few cells escaped the primary tumor by migration and invasion to target organs, so we hypothesized CXCR4 played important roles in metastasis when it translocated into the nucleus.

In the present study, we demonstrated that mutation in NLS '146RPRK149' within CXCR4 prevented its translocation to the nucleus and inhibited proliferation, migration and invasion of RCC cells, suggesting that nuclear localization of CXCR4 may be a mechanism by which RCC cells survive and spread. It indicated to us that antagonizing nuclear transport pathways or the function of nuclear CXCR4 is a rational approach for RCC therapy.

To date, increasing evidence supports that CXCR4 is highly expressed in tumor tissues and correlated with poor progression in RCC (34). However, few studies have evaluated the biological function of subcellular localization of CXCR4 in RCC cells. The present study showed that CXCR4 presented in both the nucleus and cytoplasm in RCC cells and determined the accurate location of the NLS and the impact that nuclear CXCR4 may have on RCC growth and metastasis. Our previous study suggested that 90-170 amino acid sequence within CXCR4 served as NLS (23). A prior study by Ayesha et al presumed a non-traditional NLS, '146RPRK149', within CXCR4 by PSORTII and demonstrated that removal of the putative NLS attenuated its nuclear localization in prostate cancer cells (30). We observed that nuclear localization of CXCR4 decreased obviously when arginine 146, 148 and 149 were simultaneously mutated to alanine, which indicated that the 'RPRK' motif may be involved in localization of CXCR4 to the nucleus in RCC.

Previous studies found a correlation of CXCR4 expression levels with metastatic occurrence of RCC cells, but had not shown an association of nuclear localization of CXCR4 and biological behavior of RCC cells, we then examined whether 'RPRK' motif mutation and subsequently CXCR4 subcellular localization, could alter the growth and metastatic potential of RCC cells. Our results suggested that nuclear CXCR4 was a positive regulator in the growth of RCC cells. We also found that migration, and invasion of RCC cells bearing CXCR4MutNLS were significantly repressed. Prevention of CXCR4 translocation to nucleus effectively inhibited proliferation, migration and invasion in RCC cells. This may partly explain the fact that nuclear CXCR4 was detected in many metastatic RCC patients, and the subcellular distribution of CXCR4 may play a significant role in RCC progression.

Since CXCR4 nuclear localization determined RCC progression, blockage of this process may be a novel promising therapeutic approach for RCC patients. In the present study, we found that NMMHC-IIA could particularly bind with CXCR4 and promote its nuclear translocation. NMMHC-IIA belongs to the myosin II subfamily and comprise a complex of two non-muscle myosin II heavy chains, two essential light chains and two regulatory light chains (35). NMMHC-IIA has been reported to participate in many steps of cancer metas- 
tasis (36). A previous study found that CXCR4 could bind with NMMHC-IIA in T lymphocytes(37), but its association with CXCR4 subcellular distribution was almost unknown. The present study confirmed that NMMHC-IIA could bind with CXCR4 and also promoted its nuclear translocation. Furthermore, pretreatment with blebbistatin inhibited nuclear CXCR4-induced proliferation, migration and invasion of RCC cells. This finding is consistent with previous studies demonstrating impaired migration in NMMHC-IIA-depleted MDA-MB-231 breast cancer cells (38) and blebbistatintreated pancreatic adenocarcinoma cells (39). The present study suggested that CXCR4 translocated to the nucleus and subsequently promoted RCC cell growth and metastasis partly dependent on NMMHC-IIA.

In conclusion, the data presented above provided clear evidence of CXCR4 translocated into the nucleus and acted as a functional, ligand-responsive receptor in RCC cells. Inhibition of CXCR4 nuclear localization could effectively suppress the proliferation, migration and invasion of RCC cells. Given that nuclear CXCR4 promotes metastatic ability in RCC, this investigation provided a novel mechanism to illuminate the nuclear distribution of CXCR4 as previously reported. We also demonstrated that NMMHC-IIA was functionally involved in the process of CXCR4 nuclear translocation and then affected its biology functions, possibly providing promises for new therapeutic interventions for metastatic RCC. Further investigation is still required to explore the accurate mechanism of nuclear CXCR4 promoting RCC progression and its association with clinical prognosis in RCC patients.

\section{Acknowledgements}

The present study was supported by the National Nature Science Foundation of China (nos. 81272817 and 81172447).

\section{References}

1. DeSantis CE, Lin CC, Mariotto AB, Siegel RL, Stein KD, Kramer JL, Alteri R, Robbins AS and Jemal A: Cancer treatment and survivorship statistics, 2014. CA Cancer J Clin 64: 252-271, 2014

2. An H, Xu L, Zhu Y, Lv T, Liu W, Liu Y, Liu H, Chen L, Xu J and Lin Z: High CXC chemokine receptor 4 expression is an adverse prognostic factor in patients with clear-cell renal cell carcinoma. Br J Cancer 110: 2261-2268, 2014.

3. Stewart GD, O'Mahony FC, Powles T, Riddick AC, Harrison DJ and Faratian D: What can molecular pathology contribute to the management of renal cell carcinoma? Nat Rev Urol 8: 255-265, 2011.

4. Karakiewicz PI, Briganti A, Chun FK, Trinh QD, Perrotte P, Ficarra V, Cindolo L, De la Taille A, Tostain J, Mulders PF, et al: Multi-institutional validation of a new renal cancer-specific survival nomogram. J Clin Oncol 25: 1316-1322, 2007.

5. Lam JS, Leppert JT, Figlin RA and Belldegrun AS: Role of molecular markers in the diagnosis and therapy of renal cell carcinoma. Urology 66 (Suppl 5): S1-S9, 2005.

6. Libura J, Drukala J, Majka M, Tomescu O, Navenot JM, Kucia M, Marquez L, Peiper SC, Barr FG, Janowska-Wieczorek A, et al: CXCR4-SDF-1 signaling is active in rhabdomyosarcoma cells and regulates locomotion, chemotaxis, and adhesion. Blood 100: 2597-2606, 2002.

7. Phillips RJ, Burdick MD, Lutz M, Belperio JA, Keane MP and Strieter RM: The stromal derived factor-1/CXCL12-CXC chemokine receptor 4 biological axis in non-small cell lung cancer metastases. Am J Respir Crit Care Med 167: 1676-1686, 2003.

8. Taichman RS, Cooper C, Keller ET, Pienta KJ, Taichman NS and McCauley LK: Use of the stromal cell-derived factor-1/CXCR4 pathway in prostate cancer metastasis to bone. Cancer Res 62: 1832-1837, 2002.
9. Hall JM and Korach KS: Stromal cell-derived factor 1, a novel target of estrogen receptor action, mediates the mitogenic effects of estradiol in ovarian and breast cancer cells. Mol Endocrinol 17: 792-803, 2003.

10. Kaifi JT, Yekebas EF, Schurr P, Obonyo D, Wachowiak R, Busch P, Heinecke A, Pantel K and Izbicki JR: Tumor-cell homing to lymph nodes and bone marrow and CXCR4 expression in esophageal cancer. J Natl Cancer Inst 97: 1840-1847, 2005.

11. Kim SY, Lee CH, Midura BV, Yeung C, Mendoza A, Hong SH, Ren L, Wong D, Korz W, Merzouk A, et al: Inhibition of the CXCR4/CXCL12 chemokine pathway reduces the development of murine pulmonary metastases. Clin Exp Metastasis 25: 201-211, 2008

12. Zagzag D, Krishnamachary B, Yee H, Okuyama H, Chiriboga L, Ali MA, Melamed J and Semenza GL: Stromal cell-derived factor-1alpha and CXCR4 expression in hemangioblastoma and clear cell-renal cell carcinoma: Von Hippel-Lindau loss-of-function induces expression of a ligand and its receptor. Cancer Res 65: 6178-6188, 2005.

13. D'Alterio C, Cindolo L, Portella L, Polimeno M, Consales C, Riccio A, Cioffi M, Franco R, Chiodini P, Cartenì G, et al: Differential role of CD133 and CXCR4 in renal cell carcinoma. Cell Cycle 9: 4492-4500, 2010.

14. Cho KS, Yoon SJ, Lee JY, Cho NH, Choi YD, Song YS and Hong SJ: Inhibition of tumor growth and histopathological changes following treatment with a chemokine receptor CXCR4 antagonist in a prostate cancer xenograft model. Oncol Lett 6: 933-938, 2013.

15. Teicher BA and Fricker SP: CXCL12 (SDF-1)/CXCR4 pathway in cancer. Clin Cancer Res 16: 2927-2931, 2010.

16. Na IK, Scheibenbogen C, Adam C, Stroux A, Ghadjar P, Thiel E, Keilholz U and Coupland SE: Nuclear expression of CXCR4 in tumor cells of non-small cell lung cancer is correlated with lymph node metastasis. Hum Pathol 39: 1751-1755, 2008.

17. Spano JP, Andre F, Morat L, Sabatier L, Besse B, Combadiere C, Deterre P, Martin A, Azorin J, Valeyre D, et al: Chemokine receptor CXCR4 and early-stage non-small cell lung cancer: Pattern of expression and correlation with outcome. Ann Oncol 15: 613-617, 2004.

18. Masuda T, Nakashima Y, Ando K, Yoshinaga K, Saeki H, Oki E, Morita M, Oda Y and Maehara Y: Nuclear expression of chemokine receptor CXCR4 indicates poorer prognosis in gastric cancer. Anticancer Res 34: 6397-6403, 2014.

19. Wagner PL, Hyjek E, Vazquez MF, Meherally D, Liu YF, Chadwick PA, Rengifo T, Sica GL, Port JL, Lee PC, et al: CXCL12 and CXCR4 in adenocarcinoma of the lung: Association with metastasis and survival. J Thorac Cardiovasc Surg 137: 615-621, 2009.

20. Yoshitake N, Fukui H, Yamagishi H, Sekikawa A, Fujii S, Tomita S, Ichikawa K, Imura J, Hiraishi $\mathrm{H}$ and Fujimori T: Expression of SDF-1 alpha and nuclear CXCR4 predicts lymph node metastasis in colorectal cancer. Br J Cancer 98: 1682-1689, 2008.

21. Speetjens FM, Liefers GJ, Korbee CJ, Mesker WE, van de Velde CJ, van Vlierberghe RL, Morreau H, Tollenaar RA and Kuppen PJ: Nuclear localization of CXCR4 determines prognosis for colorectal cancer patients. Cancer Microenviron 2: $1-7,2009$

22. Wang L, Chen W, Gao L, Yang Q, Liu B, Wu Z, Wang Y and Sun Y: High expression of CXCR4, CXCR7 and SDF-1 predicts poor survival in renal cell carcinoma. World J Surg Oncol 10: 212,2012

23. Wang LH, Liu Q, Xu B, Chen W, Yang Q, Wang ZX and Sun YH: Identification of nuclear localization sequence of CXCR4 in renal cell carcinoma by constructing expression plasmids of different deletants. Plasmid 63: 68-72, 2010.

24. Ma QH, Futagawa T, Yang WL, Jiang XD, Zeng L, Takeda Y, Xu RX, Bagnard D, Schachner M, Furley AJ, et al: A TAG1-APP signalling pathway through Fe65 negatively modulates neurogenesis. Nat Cell Biol 10: 283-294, 2008.

25. Lyu J, Yamamoto V and Lu W: Cleavage of the Wnt receptor Ryk regulates neuronal differentiation during cortical neurogenesis. Dev Cell 15: 773-780, 2008.

26. Carpenter G and Liao HJ: Trafficking of receptor tyrosine kinases to the nucleus. Exp Cell Res 315: 1556-1566, 2009.

27. Don-Salu-Hewage AS, Chan SY, McAndrews KM, Chetram MA, Dawson MR, Bethea DA and Hinton CV: Cysteine (C)-x-C receptor 4 undergoes transportin 1-dependent nuclear localization and remains functional at the nucleus of metastatic prostate cancer cells. PLoS One 8: e57194, 2013. 
28. Yan L, Cai Q and Xu Y: The ubiquitin-CXCR4 axis plays an important role in acute lung infection-enhanced lung tumor metastasis. Clin Cancer Res 19: 4706-4716, 2013.

29. Tripathi A, Davis JD, Staren DM, Volkman BF and Majetschak M: CXC chemokine receptor 4 signaling upon co-activation with stromal cell-derived factor- $1 \alpha$ and ubiquitin. Cytokine 65: 121-125, 2014.

30. Burgess A, Buck M, Krauer K and Sculley T: Nuclear localization of the Epstein-Barr virus EBNA3B protein. J Gen Virol 87: 789-793, 2006

31. Ren M, Qiu S, Venglat P, Xiang D, Feng L, Selvaraj G and Datla R Target of rapamycin regulates development and ribosomal RNA expression through kinase domain in Arabidopsis. Plant Physiol 155: 1367-1382, 2011.

32. Zhao FL and Guo W: Expression of stromal derived factor-1 (SDF-1) and chemokine receptor (CXCR4) in bone metastasis of renal carcinoma. Mol Biol Rep 38: 1039-1045, 2011.

33. Wang L, Wang Z, Yang B, Yang Q, Wang L and Sun Y: CXCR4 nuclear localization follows binding of its ligand SDF-1 and occurs in metastatic but not primary renal cell carcinoma. Oncol Rep 22: 1333-1339, 2009.
34. Almofti A, Uchida D, Begum NM, Tomizuka Y, Iga H, Yoshida $H$ and Sato M: The clinicopathological significance of the expression of CXCR4 protein in oral squamous cell carcinoma. Int J Oncol 25: 65-71, 2004.

35. Vicente-Manzanares M, Ma X, Adelstein RS and Horwitz AR: Non-muscle myosin II takes centre stage in cell adhesion and migration. Nat Rev Mol Cell Biol 10: 778-790, 2009.

36. Aguilar-Cuenca R, Juanes-García A and Vicente-Manzanares M: Myosin II in mechanotransduction: Master and commander of cell migration, morphogenesis, and cancer. Cell Mol Life Sci 71: 479-492, 2014

37. Carpenter G and Red Brewer M: EpCAM: Another surface-tonucleus missile. Cancer Cell 15: 165-166, 2009.

38. Hindman B, Goeckeler Z, Sierros K and Wysolmerski R: Non-muscle myosin II isoforms have different functions in matrix rearrangement by MDA-MB-231 cells. PLoS One 10: e0131920, 2015.

39. Duxbury MS, Ashley SW and Whang EE: Inhibition of pancreatic adenocarcinoma cellular invasiveness by blebbistatin: A novel myosin II inhibitor. Biochem Biophys Res Commun 313: 992-997, 2004 